\title{
Analysis of loco-regional and distant recurrences in breast cancer after conservative surgery
}

\author{
Mostafa Elsayed ${ }^{1 *}$, Mahmoud Alhussini ${ }^{2}$, Ahmed Basha $^{3}$ and A. T. Awad ${ }^{4}$
}

\begin{abstract}
Background: A number of patients treated conservatively for breast cancer will develop loco-regional and distant recurrences. Our aim was to determine how their occurrence may be linked to the evolution of the disease.

Methods: We analyzed 238 women treated by conservative breast surgery and breast irradiation in a single institution. We evaluated the prognostic factors associated with loco-regional and distant recurrences and the prognostic value of local and regional recurrences on systemic progression.

Results: After a median follow-up of 5 year (range 1-10), 16 (6.72\%) patients in the breast conservative surgery (BCS) groups had loco-regional recurrence. For distant recurrence, 10 (4.2\%) patients had experienced distant recurrence. Lympho-vascular invasion (HR 2.55; 95 \% Cl, 076 to 8.49) and an extensive intraductal component ( $\mathrm{HR}, 2.22 ; 95 \% \mathrm{Cl}$, 0.69 to 7.15$)$ and nodal status are risk factors for loco-regional recurrence (LRR) after breast conservative therapy (BCT). Tumor size, nodal status, high histologic grade, and breast cancer diagnosed at a young age ( $\leq 35$ years) are correlated with higher distant recurrence rates after BCT.

Conclusions: Risk factors for LRR after BCS include lympho-vascular invasion, extensive inraductal component, and high nodal status, where as risk factors for distant recurrence include tumor size, nodal status, high histologic grade, and breast cancer diagnosed at a young age ( $\leq 35$ years).
\end{abstract}

\section{Background}

The surgical treatment of breast cancer changed substantially over the past decades. There was a shift from the orthodox treatment applying modified radical mastectomy (MRM) to breast conservative surgery (BCS) with radiotherapy [1-4]; BCS became the standard treatment for patients with early breast cancer. It provides a better quality of life for these patients and has the same overall survival if compared with mastectomy. However, it is associated with a higher incidence of loco-regional recurrence. This event may be a biomarker of disease aggressiveness as distant spread is a frequent accompaniment [5-11].

In this study, we collected data on 238 women treated by BCS and breast radiotherapy in order to identify and assess the risk factors that might predict the occurrence

\footnotetext{
*Correspondence: m_elsayed2009@yahoo.com

${ }^{1}$ General Surgery and Surgical Oncology, Alexandria University Students

Hospital, Alexandria, Egypt

Full list of author information is available at the end of the article
}

of loco-regional and distant recurrence after BCS aiming at selecting the suitable patients with high risk of local breast recurrence after BCS.

\section{Methods}

The data of operable patients, admitted and managed at the Surgical Oncology unit, Alexandria Faculty of Medicine, between 2005 and 2014, were retrospectively reviewed and analyzed.

The data of 238 patients were included; the following were recorded:

- An ethical approval statement was taken from all cases.

- Age at diagnosis: young patients are defined as younger than 35 years.

- Tumor characteristics: size, nodal status, presence of lympho-vascular invasion, amount of intraductal component, tumor grade, margin status, hormone receptor, and Her2 neu status.

- The follow-up period of the patients was registered. 
Table 1 Age distribution

\begin{tabular}{cll}
\hline Age in years & No & $\%$ \\
$<35$ & 21 & 8.8 \\
$>35$ & 217 & 91.2 \\
Range & $24-80$ & \\
Mean \pm SD & $52.0 \pm 10.8$ & \\
\hline
\end{tabular}

- The occurrence of loco-regional recurrence or distant metastases during the follow-up period was recorded and considered as an end point for follow-up.

Local recurrence is defined as recurrence in the original tumor bed (for BCS) or field of mastectomy.

Regional recurrence refers to metastatic disease in the ipsilateral axilla or supraclavicular lymph nodes alone or in combination with the involvement of ipsilateral breast.

Loco-regional recurrence-free survival of patients who underwent BCS was estimated using the Kaplan-Meier method and compared among different categories using log-rank tests (univariable analysis of risk factors for loco-regional recurrence).

Distal recurrence-free survival for both groups will be analyzed using the same test (univariable analysis of risk factors for distal recurrence).

Associations with local recurrence after BCS were further evaluated using multivariable Cox proportional hazards regression model and summarized with hazard ratios $95 \%$ confidence intervals (CIs).

Associations with distal recurrence after BCS were further evaluated using multivariable Cox regression model.

\section{Results}

Age of 35 years or younger represented 21 (8.8\%) patients in this study. Two hundred seventeen (91.2\%) patients had an age $>35$ year. Median age was 52 years (range 2480) (Table 1).

Invasive ductal carcinoma represented 228 (95\%) of all patients which was considered the commonest

Table 2 Histopathological types and grade

\begin{tabular}{cll}
\hline Histology & No & $\%$ \\
IDC & 228 & 95 \\
ILC & 10 & 4.2 \\
Grade & & \\
I & 8 & 3.4 \\
II & 182 & 76.5 \\
III & 48 & 20.2 \\
\hline
\end{tabular}

Table 3 Tumour characteristics

\begin{tabular}{ccc}
\hline Tumour characteristics & No & $\%$ \\
\hline Size & 146 & 61.3 \\
T1 & 86 & 36.1 \\
T2 & 6 & 2.5 \\
T3 & & \\
LNs & 121 & 50.8 \\
N0 & 77 & 32.4 \\
N1 & 25 & 10.5 \\
N2 & 15 & 6.3 \\
N3 & & \\
Stage & 74 & 31.1 \\
I & 117 & 49.2 \\
II & 47 & 19.7 \\
III & &
\end{tabular}

histopathological type in this study. Grade II breast cancer patients were 182 (76.5\%) patients (Table 2).

Of the included 238 patients, 146 (61.3\%) patients presented with a clinical tumor of $2 \mathrm{~cm}$ or less.

One hundred twenty-one $(50.8 \%)$ patients had no pathological LNS.

Stage I-II breast cancer patients were 191 (80.3 \%) patients. Forty-seven $(19.7 \%)$ patients were stage III breast cancer and underwent BCS. Thirteen (27.6\%) patients had neoadjuvant chemotherapy before surgery (Tables 3 and 4).

Luminal A subtype represented 223 (93.7\%) of all patients. Triple negative subtype represented eight (3.3\%) of all patients. (Tables 5 and 6).

Histopathological examination revealed that 47 (19.7\%) patients had excess intraductal component of the tumor, whereas 58 (24.4\%) had lympho-vascular invasion.

Adjuvant radiotherapy was given to 238 (100 \%) patients.

Adjuvant chemotherapy was given to 201 (84.5\%) patients.

Hormonal therapy was given to 215 (90.3 \%) patients (Table 7).

After a median follow-up of 5 years (range $1-10$ years), $16(6.72 \%)$ patients had loco-regional recurrence (LRR), whereas 10 (4.2\%) patients had distant recurrence. Using the Kaplan-Meier method to determine the loco-

Table 4 Stage and procedure performed

\begin{tabular}{lll}
\hline Stage & No \& \% & Operation \\
\hline I\&II & $191(43 \%)$ & BCS $191(43 \%)$ \\
III & $47(21.3 \%)$ & $\begin{array}{l}\text { BCS } 47(21.3 \%) \\
\text { chemotherapy) }\end{array}$ \\
\hline
\end{tabular}


Table 5 Hormone receptors

\begin{tabular}{lll}
\hline Hormone receptors & No & $\%$ \\
\hline Estrogen & 12 & 5 \\
- ve & 24 & 10.1 \\
+ & 120 & 50.4 \\
++ & 82 & 34.5 \\
+++ & & \\
Progesterone & 19 & 8 \\
-ve & 59 & 24.8 \\
+ & 104 & 43.7 \\
++ & 56 & 23.5 \\
+++ & & \\
Her2 & 209 & 87.8 \\
- ve & 16 & 6.7 \\
+ & 1 & 0.4 \\
++ & 12 & 5 \\
+++ & & \\
\hline
\end{tabular}

regional recurrence-free survival and distant recurrencefree survival; it was found to be 92.7 and $96.4 \%$, respectively.

\section{Prognostic factors for loco-regional recurrence}

Analysis by the Cox proportional hazards model (Table 8), according to treatment actually given, demonstrated that relative risk of LRR for patients with lympho-vascular invasion compared with those without lympho-vascular invasion was 2.55 after BCS. The 5-year free survival of LRR after BCS was $89.1 \%$ for patients with lymphvascular invasion and $94 \%$ for those without lymphovascular invasion; (Fig. 1).

Patients with high nodal status have a 2.46 times higher risk of developing LRR after BCS compared with those with low nodal status.

The 5-year free survival of LRR after BCS was $75 \%$ for N3 patients and $95.7 \%$ for N1 patients (Fig. 2).

The relative risk of LRR for patients with intraductal component was 2.22 times after BCS compared with those without intraductal component. The 5-year free

Table 6 Biological subtypes

\begin{tabular}{llc}
\hline Type & No & $\%$ \\
\hline Luminal A & 223 & 93.7 \\
Luminal B & 4 & 1.7 \\
Triple negative & 8 & 3.3 \\
Her2 enriched & 3 & 1.3 \\
\hline
\end{tabular}

Table 7 Adjuvant treatment

\begin{tabular}{ll}
\hline Adjuvant treatment & Patients \\
\hline Chemotherapy & No: $37(15.5 \%)$ \\
& Yes: $201(84.5 \%)$ \\
Radiotherapy & Yes: all cases \\
Hormonal & No: $23(9.7 \%)$ \\
& Yes: $215(90.33 \%)$ \\
\hline
\end{tabular}

survival of LRR after BCS was $92.1 \%$ for patients with intraductal component and $92.8 \%$ for patients without intraductal component (Fig. 3).

The 5-year free survival of LRR after BCS was 94.4 \% for patients who received hormonal therapy and $81.3 \%$ for patients who did not receive hormonal therapy (Fig. 4).

\section{Prognostic factors for distant recurrence}

According to the results of the multivariate Cox proportional hazards survival analysis, tumor size, nodal status, and histologic grade were all highly predictor factors of distant recurrence after BCS (Table 9).

In addition, young age is an independent predictor of distant recurrence after BCS. The 5-year free survival of distant recurrence after BCS was $90.9 \%$ for patient aged 35 years and younger and $97 \%$ for patients over 35 years (Fig. 5 ).

Hormonal therapy is also a protecting factor against distant recurrence after BCS with $\mathrm{HR}=0.17$; CI $95 \%=$ 0.033-0.91. The 5-year survival of distant recurrence after BCS was $97.5 \%$ for patients who received hormonal therapy and $85.7 \%$ for those who did not receive hormonal therapy (Fig. 6).

Table 8 Cox regression for loco-regional recurrence

\begin{tabular}{|c|c|c|c|c|c|c|c|}
\hline \multicolumn{8}{|c|}{ Variables in the equation } \\
\hline \multirow[t]{2}{*}{ Operation type } & & \multirow[t]{2}{*}{ B } & \multirow[t]{2}{*}{ SE } & \multirow[t]{2}{*}{ Sig. } & \multirow[t]{2}{*}{$\mathrm{HR}$} & \multicolumn{2}{|c|}{$95.0 \% \mathrm{Cl}$ for $\mathrm{HR}$} \\
\hline & & & & & & Lower & Upper \\
\hline \multirow[t]{9}{*}{$\mathrm{BCT}$} & Age & .003 & .024 & .901 & 1.003 & .957 & 1.052 \\
\hline & $\mathrm{T}$ & .244 & .680 & .719 & 1.277 & .337 & 4.839 \\
\hline & LNs & .900 & .691 & .193 & 2.461 & .635 & 9.541 \\
\hline & Stage & -.911 & .885 & .303 & .402 & .071 & 2.277 \\
\hline & Grade & -1.322 & .603 & .028 & .267 & .082 & .870 \\
\hline & IDC & .799 & .596 & .180 & 2.223 & .691 & 7.151 \\
\hline & Invasion & .938 & .613 & .126 & 2.555 & .768 & 8.497 \\
\hline & Postop_chemo & -1.137 & .745 & .127 & .321 & .074 & 1.382 \\
\hline & Horm & -1.858 & .658 & .005 & .156 & .043 & .567 \\
\hline
\end{tabular}

$\mathrm{HR}>1$ is considered as a risk factor 


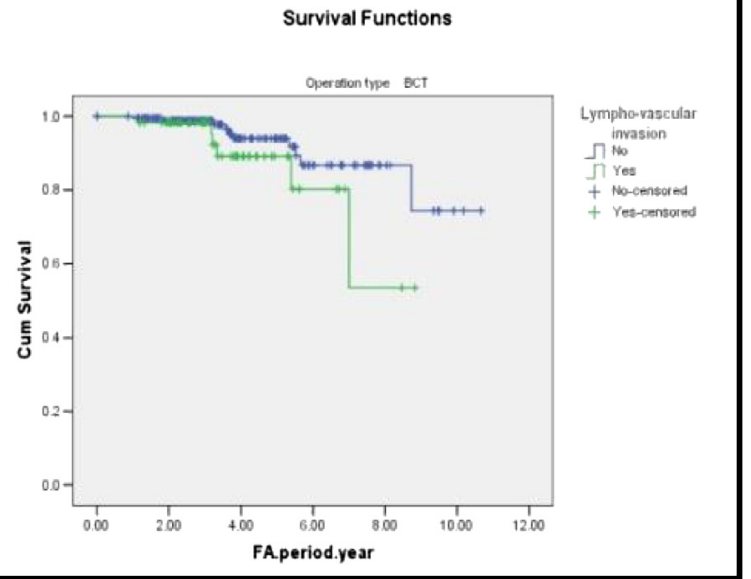

Fig. 1 A Kaplan-Meier plot showing LRR-free survival by lymphovascular invasion in BCS group

\section{Discussion}

The surgical treatment of breast cancer has been changed during the previous decades towards a less extensive surgery. Breast conservative surgery (BCS) is a model of this type of surgery which in properly selected patients provides local control of the disease. Based on the systemic disease concept of cancer breast, the removal of the primary does not obviate the risk of distant spread. Thus, our concern in the present study was to analyze the risk factors associated with loco-regional recurrence after BCS as local recurrence will obviate the purpose of breast conservation $[12,13]$.

The study revealed that the presence of an extensive intraductal component (EIC), lympho-vascular invasion

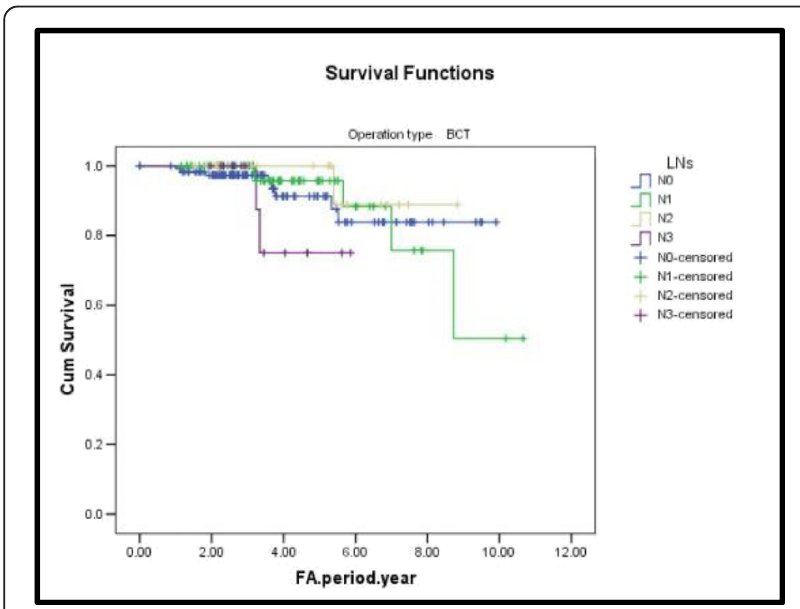

Fig. 2 A Kaplan-Meier plot showing LRR-free survival by nodal status in BCS group

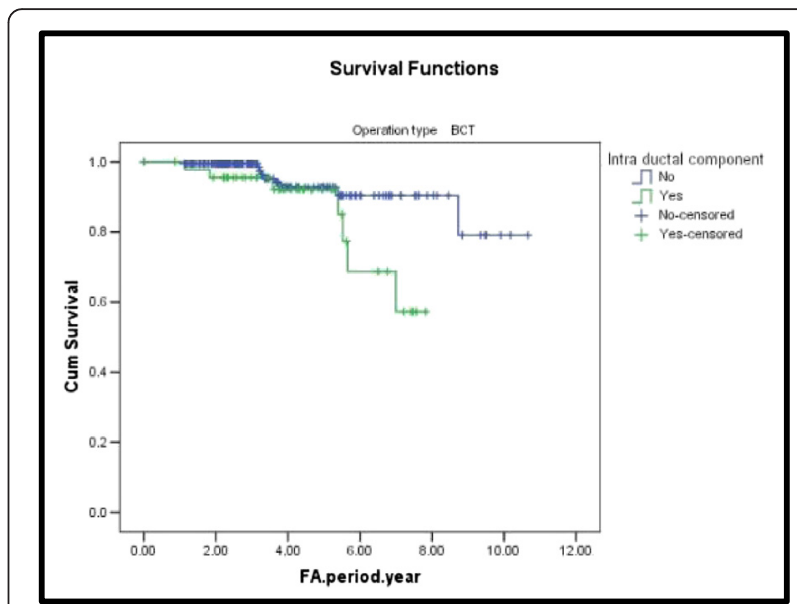

Fig. 3 A Kaplan-Meier plot showing LRR-free survival by intraductal component in BCS group

and nodal status are associated with an increased risk of LRR after BCS.

EIC is an established risk factor for LRR after breast conservative therapy (BCT) [5, 14-16]. Invasive breast carcinoma is accompanied by an extensive component of DCTS in $15-30 \%$ of patients.

DCIS grows along the ducts in the breast without invasion of the underlying tissue, which results in a nonpalpable lesion difficult to remove with tumor-free margins. When EDCIs are completely removed with negative tumor margins, it loses its predicative value for LRR [5, 14-19]. In a pooled analysis of the EORTC 10801 and the DBCG 82 TM trials, lympho-vascular invasion causes a higher risk of LRR after BCT [20].

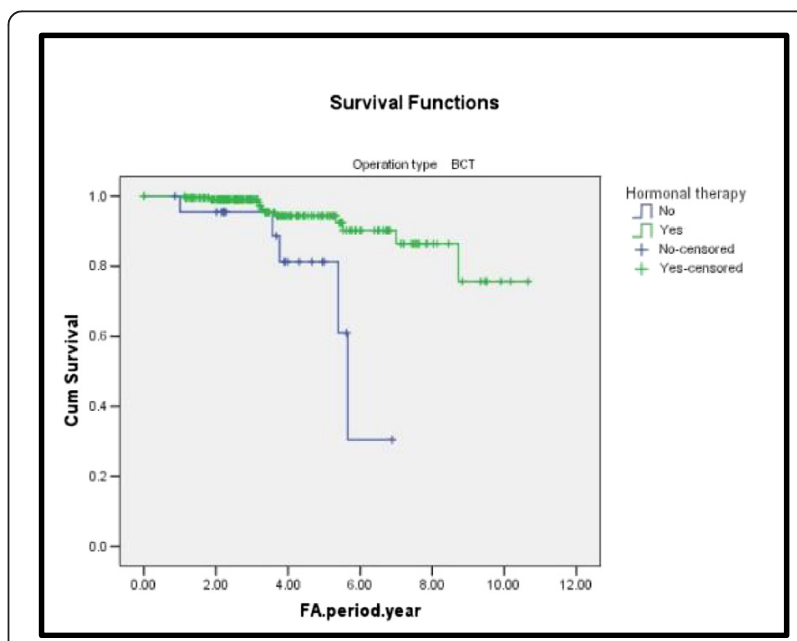

Fig. 4 A Kaplan-Meier plot showing LRR-free survival by hormonal therapy in BCS group 
Table 9 Cox regression for distal recurrence

\begin{tabular}{|c|c|c|c|c|c|c|c|}
\hline \multicolumn{8}{|c|}{ Variables in the equation } \\
\hline \multirow[t]{2}{*}{ Operation type } & & \multirow[t]{2}{*}{ B } & \multirow[t]{2}{*}{ SE } & \multirow[t]{2}{*}{ Sig. } & \multirow[t]{2}{*}{$\mathrm{HR}$} & \multicolumn{2}{|c|}{$95.0 \% \mathrm{Cl}$ for $\mathrm{HR}$} \\
\hline & & & & & & Lower & Upper \\
\hline \multirow[t]{9}{*}{ BCT } & Age & -.014 & .036 & .684 & .986 & .919 & 1.057 \\
\hline & $\mathrm{T}$ & .897 & .785 & .253 & 2.453 & .527 & 11.417 \\
\hline & LNs & .656 & 635 & .302 & 1.927 & .555 & 6.692 \\
\hline & Stage & .065 & .947 & .945 & 1.067 & .167 & 6.825 \\
\hline & Grade & .392 & .744 & .598 & 1.480 & .344 & 6.356 \\
\hline & IDC & .132 & .776 & .864 & 1.142 & .250 & 5.221 \\
\hline & Invasion & -1.233 & .949 & .194 & .291 & .045 & 1.870 \\
\hline & Postop_XRT & -.293 & 1.214 & .809 & .746 & .069 & 8.056 \\
\hline & Horm & -1.759 & .848 & .038 & .172 & .033 & .907 \\
\hline
\end{tabular}

$\mathrm{HR}>1$ is considered as a risk factor

This result is also concordant with the observations made by Salim Alrahbi et al., Zahra MA Mohammed et al., and Bent Ejlertsen et al. [21-23].

In agreement with others, positive nodal status is a predictor for LRR in patients of BCT group [24]. Our study suggests a favorable effect of postoperative radiotherapy and adjuvant systemic treatment on LRR and distant metastases for BCS [12, 13, 20]. Today, the widespread use of adjuvant systemic therapy (chemotherapy and endocrine therapy) for both nodepositive and node-negative breast cancer, coupled with improvements in the mammographic and pathological assessment of patients undergoing breast-conserving surgery, has resulted in decreased incidence of local failure $[25,26]$.

A study in Japan investigating the occurrence of ipsilateral breast recurrence, after long-term follow-up of patients with early breast cancer, after breastconservative surgery, found it to be significantly

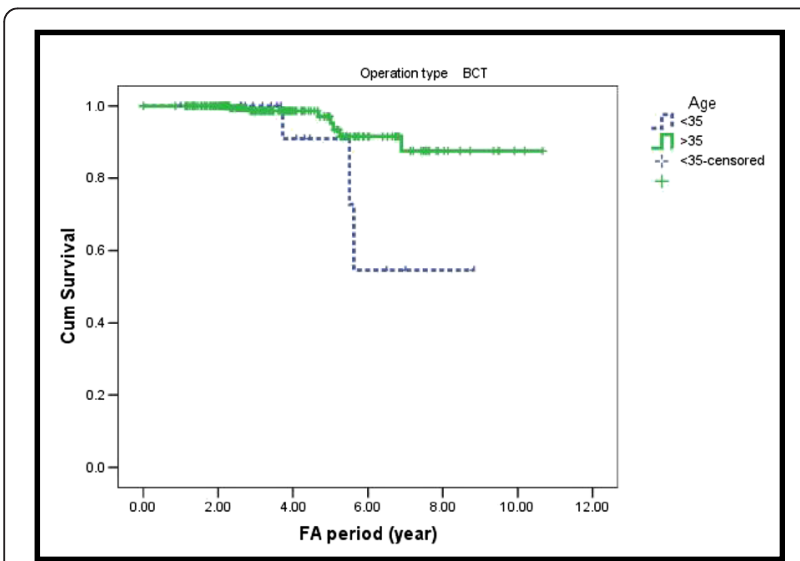

Fig. 5 A Kaplan-Meier plot showing distant recurrence-free survival by age in BCS group

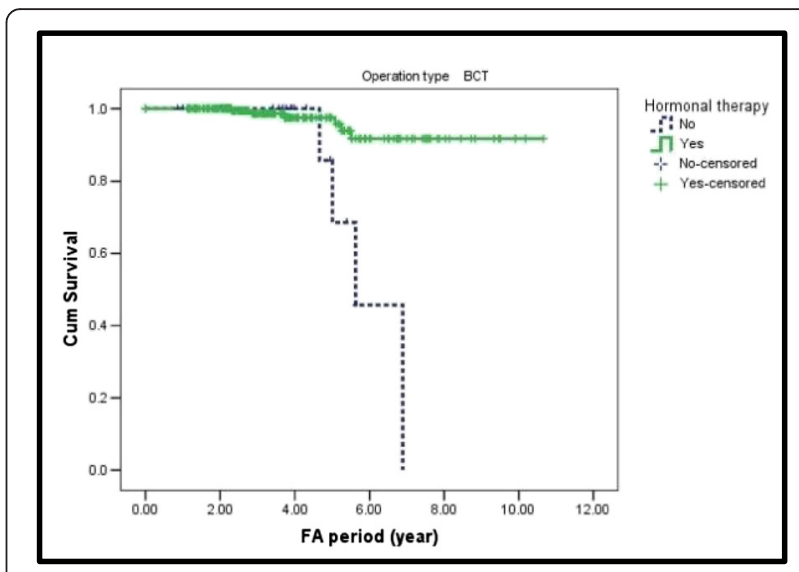

Fig. 6 A Kaplan-Meier plot showing distant recurrence-free survival by hormonal therapy in BCS group

associated with young age, positive surgical margin, and omission of radiation therapy [27].

Our study reveals that tumor size, nodal status, and high histologic grade are predictors for distant recurrence after BCS. Various studies have described an increased rate of distant metastases among those patients $[14,20,28-33]$.

Patients 35 years of age or younger appeared to have an increased risk of distant disease. The increased risk was much more prominent after BCT.

The adverse effect of young age on prognosis has been noted in several other studies and suggests that breast cancer in younger women is biologically more aggressive disease, possibly requiring more aggressive initial treatment. The question arises whether LRR might be a source of distant spread in some patients in the youngest age group. Unfortunately, numbers in the current study did not allow us to find the answer to this question $[17,34,35]$.

\section{Conclusions}

Risk factors for loco-regional recurrence and distant metastases play an important role in the decision for the treatment of breast cancer. This decision-making can be optimized if patients at high risk for loco-regional recurrence can be identified.

- Lympho-vascular invasion, EIC, and high nodal status are risk factors for LRR after BCT.

- Tumor size, nodal status, high histologic grade, and breast cancer diagnosed at a young age ( $<35$ years) are correlated with higher distant recurrence rates after BCT.

- Radiotherapy and hormonal therapy have a great role in decreasing the development of recurrence after BCS. 


\section{Competing interests}

The authors declare that they have no competing interests.

\section{Authors' contributions}

All authors read and approved the final manuscript.

\section{Author details}

${ }^{1}$ General Surgery and Surgical Oncology, Alexandria University Students Hospital, Alexandria, Egypt. ${ }^{2}$ General Surgery and Surgical Oncology, Faculty of Medicine, Alexandria University, Alexandria, Egypt. ${ }^{3}$ General Surgery and Surgical Oncology, Alexandria, Egypt. ${ }^{4}$ Surgery Department, Faculty of Medicine, Alexandria University, Alexandria, Egypt.

\section{Received: 27 January 2016 Accepted: 21 April 2016}

\section{Published online: 14 May 2016}

\section{References}

1. Fisher B, Anderson S, Bryant J, et al. Twenty-year follow-up of a randomized trial comparing total mastectomy, lumpectomy, and lumpectomy plus irradiation for the treatment of invasive breast cancer. N Engl J Med. 2002; 347:1233-41.

2. Veronesi U, Cascinelli N, Mariani L, et al. Twenty-year follow-up of a randomized study comparing breast-conserving surgery with radical mastectomy for early breast cancer. N Engl J Med. 2002;347:1227-32.

3. Goh BK, Yong WS. Eighteen-year results in the treatment of early breast carcinoma with mastectomy versus breast conservation therapy. Cancer. 2004;100:1766.

4. van Dongen JA, Voogd AC, Fentiman IS, et al. Long-term results of a randomized trial comparing breast-conserving therapy with mastectomy: European Organization for Research and Treatment of Cancer 10801 trial. J Natl Cancer Inst. 2000;92:1143-50.

5. Komoike $Y$, Akiyama $F$, lino $Y$, et al. Ipsilateral breast tumor recurrence (IBTR) after breast-conserving treatment for early breast cancer: risk factors and impact on distant metastases. Cancer. 2006;106:35-41.

6. Wapnir IL, Anderson SJ, Mamounas EP, et al. Prognosis after ipsilateral breast tumor recurrence and locoregional recurrences in five National Surgical Adjuvant Breast and Bowel Project node-positive adjuvant breast cancer trials. J Clin Oncol. 2006;24:2028-37.

7. Mamounas EP. Ipsilateral breast tumor recurrence after lumpectomy: is it time to take the bull by the horns? J Clin Oncol. 2001;19:3798-800.

8. Shen J, Hunt KK, Mirza NQ, et al. Predictors of systemic recurrence and disease-specific survival after ipsilateral breast tumor recurrence. Cancer. 2005;104:479-90.

9. de Bock GH, Putter $\mathrm{H}$, Bonnema J, et al. The impact of loco-regional recurrences on metastatic progression in early-stage breast cancer: a multistate model. Breast Cancer Res Treat. 2009;117:401-8.

10. Punglia RS, Morrow M, Winer EP, Harris JR. Local therapy and survival in breast cancer. N Engl J Med. 2007:356:2399-405.

11. Anderson SJ, Wapnir I, Dignam JJ, et al. Prognosis after ipsilateral breast tumor recurrence and locoregional recurrences in patients treated by breast-conserving therapy in five National Surgical Adjuvant Breast and Bowel Project protocols of node-negative breast cancer. J Clin Oncol. 2009; 27:2466-73.

12. Ziogas D, Roukos DH. Genetics and personal genomics for personalized breast cancer surgery: progress and systems biology. Ann Surg. 2009; 249:1067-8

13. Roukos DH. Radiation therapy for breast cancer. N Engl J Med. 2009:360:1362

14. Kim KJ, Huh SJ, Yang JH, et al. Treatment results and prognostic factors of early breast cancer treated with a breast conserving operation and radiotherapy. Jpn J Clin Oncol. 2005;35:126-33.

15. Beadle BM, Woodward WA, Buchholz TA. The impact of age on outcome in early-stage breast cancer. Semin Radiat Oncol. 2011;21:26-34.

16. Miles RC, Gullerud RE, Lohse CM, et al. Local recurrence after breast-conserving surgery: multivariable analysis of risk factors and the impact of young age. Ann Surg Oncol. 2012;19:1153-9.

17. Jones HA, Antonini N, Hart AA, et al. Impact of pathological characteristics on local relapse after breast-conserving therapy: a subgroup analysis of the EORTC boost versus no boost trial. J Clin Oncol. 2009;27:4939-47.

18. Werkhoven $\mathrm{E}$, Hart G, Tinteren $\mathrm{H}$, et al. Nomogram to predict ipsilateral breast relapse based on pathology review from the EORTC 22881-10882 boost versus no boost trial. Radiother Oncol. 2011;100:101-7.
19. Bernardi S, Bertozzi S, Londero AP, et al. Influence of surgical margins on the outcome of breast cancer patients: a retrospective analysis. World J Surg. 2014;38:2279-87.

20. Voogd AC, Nielsen M, Peterse $J$, et al. Differences in risk factors for local and distant recurrence after breast-conserving therapy or mastectomy for stage I and II breast cancer. Pooled results of two large European randomized trials. J Clin Oncol. 2001;19:1688-97.

21. Alrahbi S, Chan PM, Ho BC, Seah MD, Chen JJ, Tan EY. Extent of margin involvement, lymphovascular invasion, and extensive intraductal component predict for residual disease after wide local excision for breast cancer. Clin Breast Cancer. 2015;15:219-26.

22. Mohammed ZM, McMillan DC, Edwards J, Mallon E, Doughty JC, Orange C, et al. The relationship between lymphovascular invasion and angiogenesis, hormone receptors, cell proliferation and survival in patients with primary operable invasive ductal breast cancer. BMC Clin Pathol. 2013;13:31.

23. Ejlertsen B, Jensen MB, Rank F, Rasmussen BB, Christiansen P, Kroman N, et al. Population-based study of peritumoral lymphovascular invasion and outcome among patients with operable breast cancer. J Natl Cancer Inst. 2009:101:729-35.

24. Van Dongen JA, Bartelink H, Fentiman IS, et al. Factors influencing local relapse and survival and results of salvage treatment after breast-conserving therapy in operable breast cancer: EORTC trial 10801, breast conservation compared with mastectomy in TNM stage I and II breast cancer. Eur J Cancer. 1992;28A:801-5.

25. Nielsen HM, Overgard M, Grau C, et al. Loco-regional recurrence after mastectomy in high-risk breast cancer-risk and prognosis. An analysis of patients from the DBCG 82 b\&c randomization trials. Radiother Oncol. 2006; 79:147-55.

26. Litière $S$, Werutsky $G$, Fentiman IS, et al. Breast conserving therapy versus mastectomy for stage I-II breast cancer: 20 year follow-up of the EORTC 10801 phase 3 randomised trial. Lancet Oncol. 2012;13:412-9.

27. Ohsumi S, Sakamoto G, Takashima S, Koyama H, Shin E, Suemasu K, et al. Long-term results of breast-conserving treatment for early-stage breast cancer in Japanese women from multicenter investigation. Jpn J Clin Oncol. 2003;33:61-7.

28. Mechera R, Viehl CT, Oertli D. Factors predicting in-breast tumor recurrence after breast-conserving surgery. Breast Cancer Res Treat. 2009;116:171-7.

29. Lundin J, Lundin M, Holli K, et al. Omission of histologic grading from clinical decision making may result in overuse of adjuvant therapies in breast cancer: results from a nationwide study. J Clin Oncol. 2001;19:28-36.

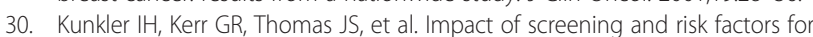
local recurrence and survival after conservative surgery and radiotherapy for early breast cancer: results from a large series with long-term follow-up. Int J Radiat Oncol, Biol, Phys. 2012;83:829-38.

31. Kato T, Kameoka S, Kimura T, et al. Angiogenesis as a predictor of long-term survival for 377 Japanese patients with breast cancer. Breast Cancer Res Treat. 2001;70:65-74.

32. Choi WW, Lewis MM, Lawson D, et al. Angiogenic and lymphangiogenic microvessel density in breast carcinoma: correlation with clinicopathologic parameters and VEGF-family gene expression. Mod Pathol. 2005;18:143-52.

33. Erdem O, Dursun A, Coşkun U, et al. The prognostic value of p53 and cerbB-2 expression, proliferative activity and angiogenesis in node-negative breast carcinoma. Tumori. 2005;91:46-52.

34. Martinez-Ramos D, Escrig J, Torrella A, et al. Risk of recurrence of nonmetastatic breast cancer in women under 40 years: a population-registry cancer study in a European country. Breast J. 2012;18:118-23.

35. van der Leij F, Elkhuizen $\mathrm{PH}$, Bartelink $\mathrm{H}$, et al. Predictive factors for local recurrence in breast cancer. Semin Radiat Oncol. 2012;22:100-7. 